Presented at the Fifth International Conference on Calorimetry, BNL, Sept. 25 - Oct 1, 1994

\title{
Radiation Effects on Front-End Electronics for Noble Liquid Calorimetry*
}

Mauro Citterio, Sergio Rescia, and Veljko Radeka

Brookhaven National Laboratory, Upton, NY 11973 USA

\begin{abstract}
DISCLAIMER
This report was prepared as an account of work sponsored by an agency of the United States Government. Neither the United States Government nor any agency thereof, nor any of their employees, makes any warranty, express or implied, or assumes any legal liability or responsibility for the accuracy, completeness, or usefulness of any information, apparatus, product, or process disclosed, or represents that its use would not infringe privately owned rights. Reference herein to any specific commercial product, process, or service by trade name, trademark, manufacturer, or otherwise does not necessarily constitute or imply its endorsement, recommendation, or favoring by the United States Government or any agency thereof. The views and opinions of authors expressed herein do not necessarily state or reflect those of the United States Government or any agency thereof.
\end{abstract}

December 1994

*This research was supported by the U.S Department of Energy:

Contract No. DE-AC02-76CH00016 


\section{DISCLAIMER}

Portions of this document may be illegible in electronic image products. Images are produced from the best available original document. 


\title{
Radiation Effects On Front-End Electronics For Noble Liquid Calorimetry*
}

\author{
Mauro Citterio, Sergio Rescia and Veljko Radeka \\ Brookhaven National Laboratory, Upton, NY 11973 USA
}

\begin{abstract}
Front-end electronics for liquid ionization chamber calorimetry at hadron collider experiments may be exposed to substantial levels of ionizing radiation and neutron fluences in a cryogenic environment. Measurements of devices built with rad-hard technologies have shown that devices able to operate in these conditions exist.

Si-JFETs and GaAs MESFET devices have been irradiated and tested at a stable cryogenic temperature up to doses of $55 \mathrm{Mrad}$ of ionizing radiation and up to neutron fluences of $4 \times 10^{14} \mathrm{n} / \mathrm{cm}^{2}$. Radiation effects on DC characteristics and on noise will be presented.
\end{abstract}

\section{Introduction}

Fast calorimetry for hadron collider experiments will have their front-end electronics mounted on the electrodes operating in a cryogenic environment ( $90 \mathrm{~K}$ for a liquid Argon calorimeter or $120 \mathrm{~K}$ for a liquid Krypton) [1].

Simulations of radiation levels show that at this experimental location the electronics might be exposed to level of ionizing radiation of $10 \mathrm{kGy}(1 \mathrm{Mrad})$ and neutron fluences up to $2 \times 10^{14} \mathrm{n} / \mathrm{cm}^{2}$ during ten years of operation at a luminosity of $2 \times 10^{34} \mathrm{~cm}^{-2} \mathrm{~s}^{-1}[2,3]$.

To evaluate the radiation effects in this regime of operation, special cryostats which allow cryogenic irradiation and DC and AC testing of active devices have been built.

Some commercially available Si-JFET and GaAs MESFETs have been irradiated under bias and monitored over several months at a steady cryogenic temperature. Several devices from each technology have been selected for identical characteristics and subdivided in two groups. A first group has been exposed to gamma rays using a $\mathrm{Co}^{60}$ source at a temperature of $90 \mathrm{~K}$. A second set of devices has been irradiated with high energy neutrons at a temperature of $98 \mathrm{~K}$ using a spallation source, protons against a $\mathrm{Li}$ target.

At the end of the tests, a total dose up to $55 \mathrm{Mrad}$ and a neutron fluence up to $4 \times 10^{14}$ $\mathrm{n} / \mathrm{cm}^{2}$ have been achieved.

The goal of this research has been not only the assessment of the radiation hardness of Si-JFET and GaAs MESFETs for cryogenic operations but also the achievement of a better understanding of the physical mechanism involved with radiation induced effects in these active devices.

Radiation effects on noise power density (measured up to frequencies of $10-30 \mathrm{MHz}$ ), equivalent noise charge (ENC) and DC characteristics of the different technologies will be presented.

- This research was supported by the U.S. Department of Energy: Contract No. DE-AC02-76CH00016. 


\section{Irradiation Tests}

The ionizing radiation environment in which the electronics will operate in the experiments has been reproduced using a $20 \mathrm{KCi} \mathrm{Co}^{60}$ gamma ray source at Brookhaven National Laboratory. The photons emitted by a $\mathrm{Co}^{60}$ source (with energy of 1.1 and $1.3 \mathrm{MeV}$ ) represent a reasonable approximation of the spectrum of the albedo photons within a typical calorimeter configuration in which the photon spectrum decreases rapidly with energy and has a characteristic energy of $\sim 1 \mathrm{MeV}$ [4].

The large neutron fluences have been simulated using a fast neutron irradiation facility at the 5.5 MV CN Van de Graaff accelerator, University of Massachusetts, Lowell [5]. This irradiation facility uses a ${ }^{7} \mathrm{Li}(\mathrm{p}, \mathrm{n})^{7} \mathrm{Be}$ reaction to produce neutrons in the $0-2 \mathrm{MeV}$ energy interval $(<E>=1 \mathrm{MeV})$, close to the neutron energy spectrum expected in the experimental conditions [4] and contains minimal portions of thermal and near thermal neutrons. The physical characteristics of the source are summarized in Table 1.

\begin{tabular}{|c|c|}
\hline Reaction type & ${ }^{7} \mathrm{Li}(p, n)^{7} \mathrm{Be}$ \\
\hline Proton beam energy & $4 \mathrm{MeV}$ \\
\hline Proton beam current & $-25 \mu \mathrm{A}$ \\
\hline Neutron fluence & $\sim 10^{14} \mathrm{n} / \mathrm{cm}^{2}$ per day \\
\hline Neutron energy spectra & $\begin{array}{l}0-2 \mathrm{MeV} \\
\langle\mathrm{E}\rangle=1.1 \mathrm{MeV}\end{array}$ \\
\hline Slow neutrons & $\begin{array}{l}(\mathrm{E}<10 \mathrm{KeV}) \\
<0.05 \%\end{array}$ \\
\hline$\gamma$-ray contamination & $\sim 10 \mathrm{Krad} /$ day \\
\hline Neutron Dosimetry & $\begin{array}{l}\text { - }{ }^{7} \mathrm{Be} \text { target activity } \\
\text { - Ni-foil activation as } \\
\text { described by ASTM E264-87 }\end{array}$ \\
\hline
\end{tabular}

Table 1. Neutron Irradiation Facility Characteristics.

This neutron source was selected because it is practically free from any gamma ray background so that neutron effects alone could be studied.

Both the irradiation facilities allowed to carry out the irradiation studies at cryogenic temperatures, however they did not give the possibility to measure on-line the characteristics of the devices under tests. Transient effects during radiation were not investigated. 


\section{Gamma ray induced effects}

\section{A. Silicon JFETs}

Silicon JFETs are remarkably radiation hard with respect to $\gamma$-rays. DC characteristics variations are hardly measurable up to $55 \mathrm{Mrad}$. Even the gate current, that had been generally observed to increase with radiation at room temperature, remained constant at $90 \mathrm{~K}$.

The only sensitive parameter to ionizing radiation is noise.

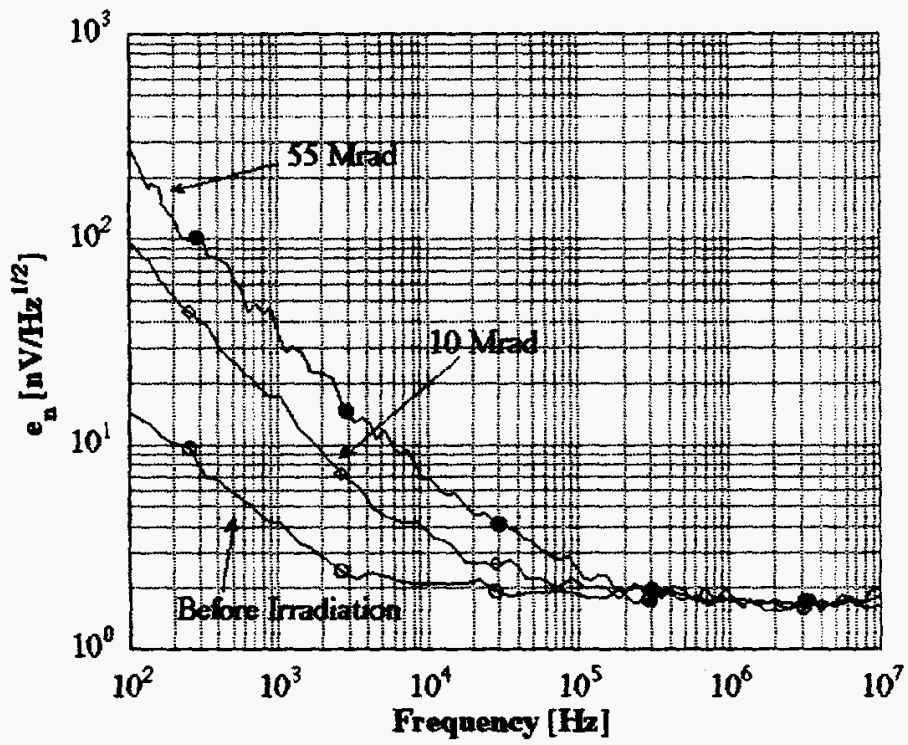

Fig. 1 Series noise spectral density at $90 \mathrm{~K}$ for a Interfet Si-NJFET exposed to $\mathrm{Co}^{60} \gamma$-rays as a function of dose. Device parameters are: $\mathrm{W} / \mathrm{L}=2500 / 3, \mathrm{~V}_{\mathrm{DS}}=2.5 \mathrm{~V}, \mathrm{I}_{\mathrm{D}}=1 \mathrm{~mA}$.

Fig. 1 shows that the spectral noise density level in the white noise region is unaffected by $\gamma$-rays. Increased noise in the low frequency region minimally degrades the signal to noise ratio since the frequency range at the fast shaping time needed for calorimetry at hadron colliders is in the $1-20 \mathrm{MHz}$ region.

The noise increase at low frequencies could be attributed to radiation induced single point defects in the device channel which act as generation-recombination centers for the majority carriers. The drain current fluctuations due to these centers can be represented as a sum of lorentzian terms in the series noise power density $e_{n}[5,6]$ :

$$
\frac{\overline{d e_{n}^{2}}}{d f}=\sum_{i=1}^{N} \frac{a_{L i}}{1+\omega^{2} \tau_{L i}^{2}}
$$

Each term is constant up to the characteristic frequency $f_{L i}=1 / 2 \pi \tau_{L i}$ and then rolls off as $1 / f^{2}$; the physical nature of the defect is described by the two constants $a_{L i}$ and $\tau_{L i}$

A measurement of the series noise spectral density versus temperature after a total dose of $55 \mathrm{Mrad}$ of ionizing radiation has been performed. A typical result of this measurement at the temperatures of $90 \mathrm{~K}$ and $120 \mathrm{~K}$ is shown in Fig. 2. 
In silicon JFETs the lorentzian noise components before irradiation are negligible at frequencies above a few hundred $\mathrm{Hz}$, at any temperature.

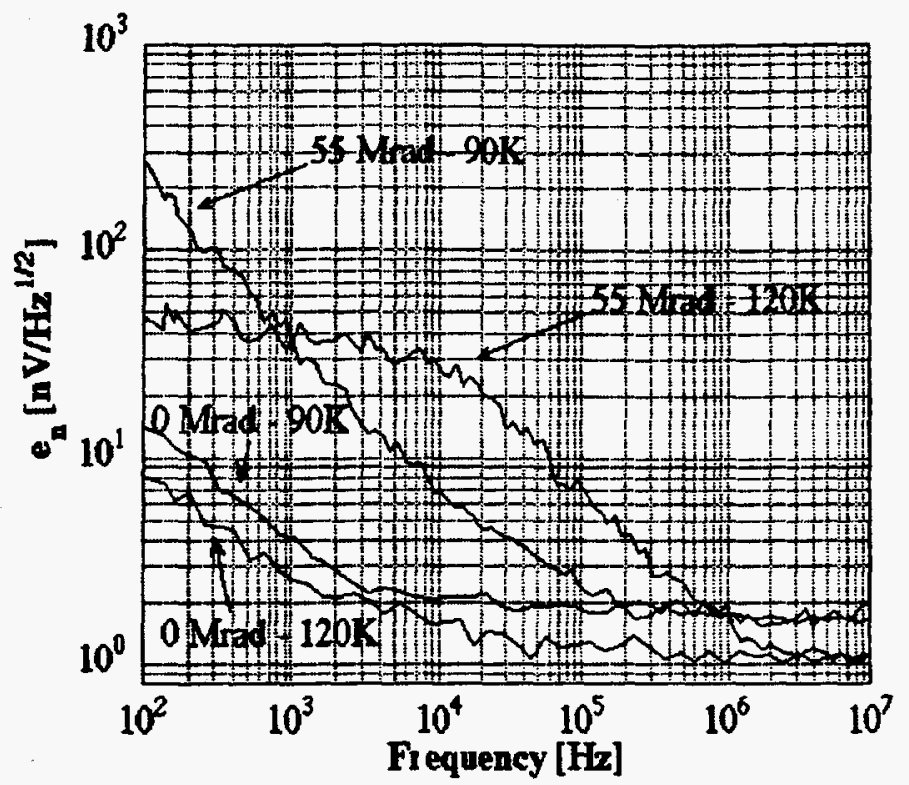

Fig. 2 Series noise spectral density at $90 \mathrm{~K}$ and $120 \mathrm{~K}$ for an Interfet Si-NJFET exposed to $\mathrm{CO}^{60} \gamma$-rays before and after irradiation. Device parameters are: $\mathrm{W} / \mathrm{L}=2500 / 3, \mathrm{~V}_{\mathrm{DS}}=2.5 \mathrm{~V}, \mathrm{~L}_{\mathrm{D}}=1 \mathrm{~mA}$.

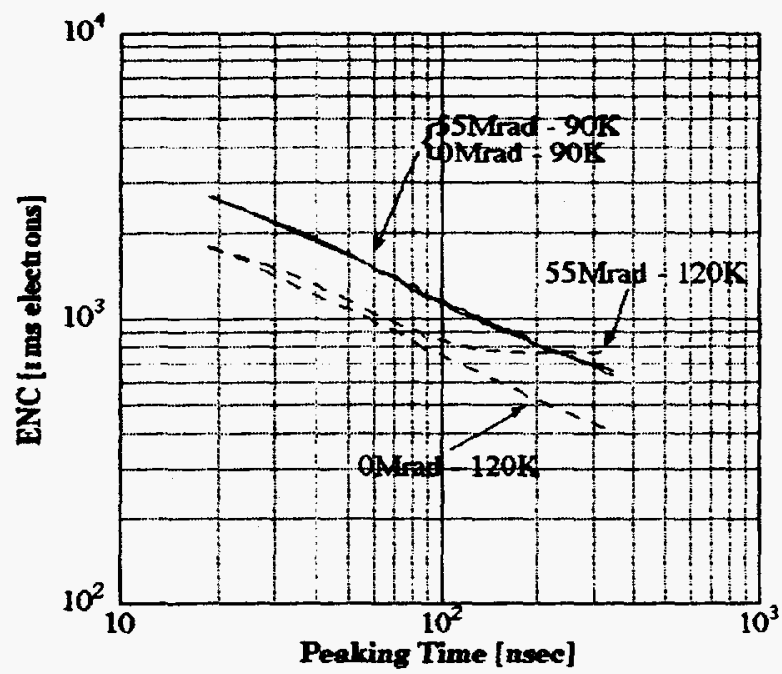

Fig. 3 Computed Equivalent Noise charge at 90K and 120K for an interfet Si-NJFET exposed to $\mathrm{Co}^{60} \gamma$-rays before and after irradiation. Device parameters are: $\mathrm{W} / \mathrm{L}=2500 / 3, \mathrm{~V}_{\mathrm{DS}}=2.5 \mathrm{~V}$, $I_{D}=1 \mathrm{~mA}, C_{\mathrm{i}=} \sim 10 \mathrm{pF}$. For the calculation: $C_{\mathrm{D}}=33 \mathrm{pF}$, no parallel noise contribution.

After gamma rays irradiation these noise contributions are enhanced and become the dominant component over a broader frequency range especially as the temperature increases. In the $90 \mathrm{~K}$ to $120 \mathrm{~K}$ temperature range the increase in noise can be attributed to one radiation 
generated lorentzian component with characteristic frequency lower than $100 \mathrm{~Hz}$ at $90 \mathrm{~K}$ and of $\sim 10 \mathrm{kHz}$ at $120 \mathrm{~K}$.
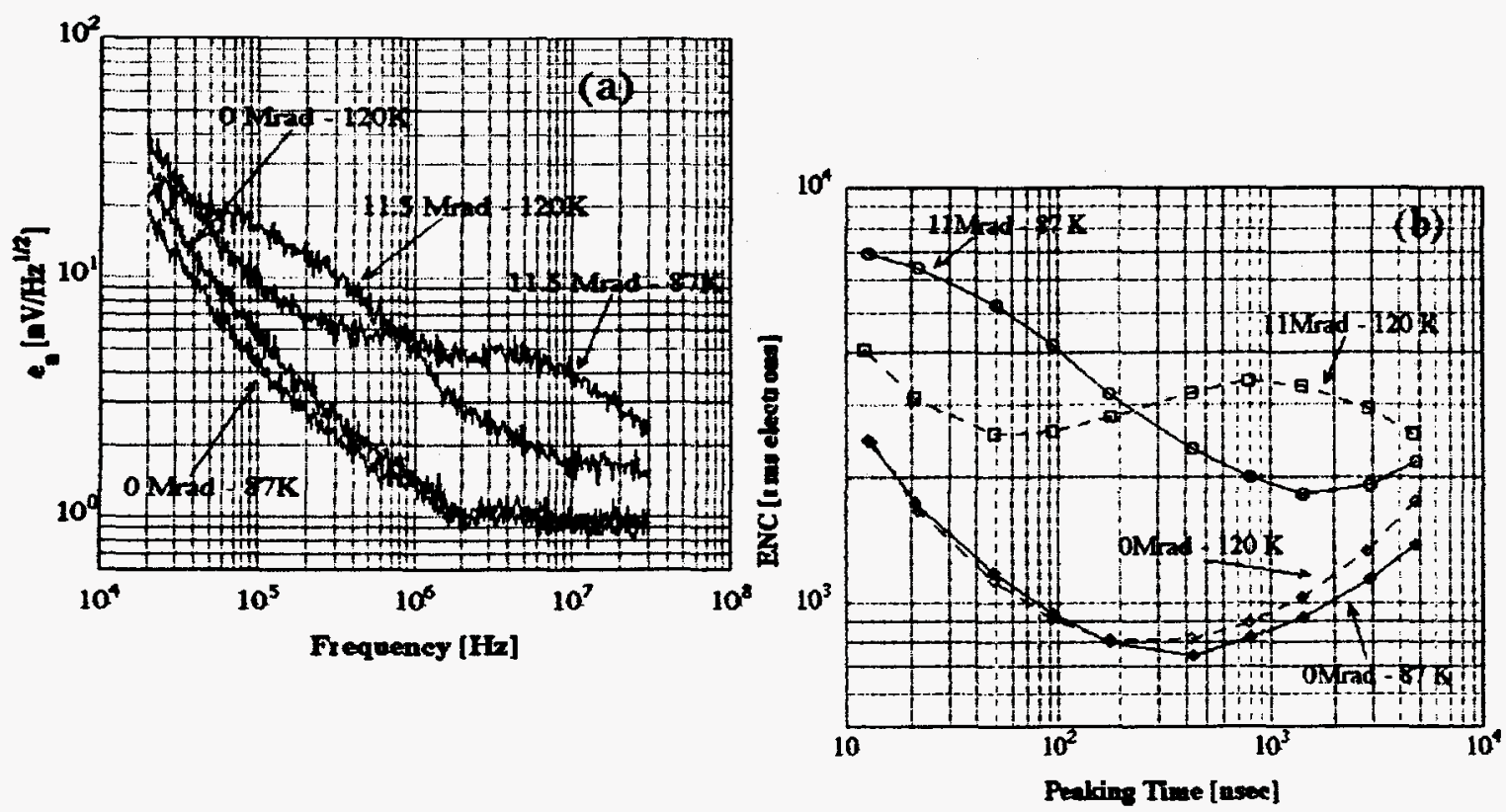

Fig. 4 Series noise spectral density (a) and equivalent noise charge (b) at $87 \mathrm{~K}$ and at $120 \mathrm{~K}$ for the input device of a GaAs preamplifier (Triquint process) exposed to $\mathrm{Co}^{60} \gamma$-rays before and after irradiation. The bias current in the device is $2 \mathrm{~mA}$ approximately.

At short processing times the noise degradation is still negligible even at $120 \mathrm{~K}$. A computed ENC in the case of a bipolar shaping, obtained from the series noise spectral density data of fig. 2 is shown in Fig. 3.

\section{B. GaAs MESFETs}

No degradation has been observed in the DC characteristics of GaAs devices up to the total dose of $55 \mathrm{Mrad}$.

GaAs MESFETs experience a larger increase in noise than Si-JFETs after $\mathrm{Co}^{60}$ exposure. Spectral noise density data show that the increase in noise is not limited to the low frequency portion of the spectra.

The radiation induced noise can be described even for GaAs MESFETs by lorentzian terms as in equation (1). The analysis of the noise versus temperature after irradiation shows that at least two lorentzian contributions, with characteristic frequencies in the region of interest, degrade the noise performances at liquid Argon and liquid Krypton temperatures (see Fig. 4a).

This hypothesis is confirmed by the analysis of ENC measurements, shown in Fig. 4b, in which the strong temperature dependency of this induced noise is shown.

The absolute noise variation as a function of dose varies with the manufacturing process and even with different runs of the same process. The radiation induced noise increase 
varies from a best case of $10-20 \%$ (NEC, Triquint) to a worst case of $200 \%$ (Triquint) at $90 \mathrm{~K}$ at $40 \mathrm{nsec}$ peaking time after a total dose of $2 \mathrm{Mrad}$.

\section{Annealing}

No annealing has been observed at cryogenic temperatures over a period of several months from the conclusion of the gamma ray irradiation for the three different technologies. No recovery in the properties has been observed even after several thermal cycles between $90 \mathrm{~K}$ and $350 \mathrm{~K}$.

\section{Neutron Induced Effects}

\section{A. Silicon JFETs}

Silicon JFETs are affected by neutrons. The lattice damage in the crystal structure generated by the neutrons affects the DC characteristics of JFETs: the pinch-off voltage as well as $I_{\text {Dss }}$ are reduced due to the generation of acceptor-like state in the channel and the deactivation of donor atoms. The trasconductance decreases too, by about $25 \%$ at constant current at $4 \times 10^{14} \mathrm{n} / \mathrm{cm}^{2}$ as shown in Fig. 5 .

The variation of noise as a function of the fluence is shown in fig. 6 . The noise increases in the low frequency region of the spectrum and the reduction in the trasconductance causes an increase in the white noise region. This degradation in the noise translates in a 30-35\% increase in ENC at 40 nsec shaping time.

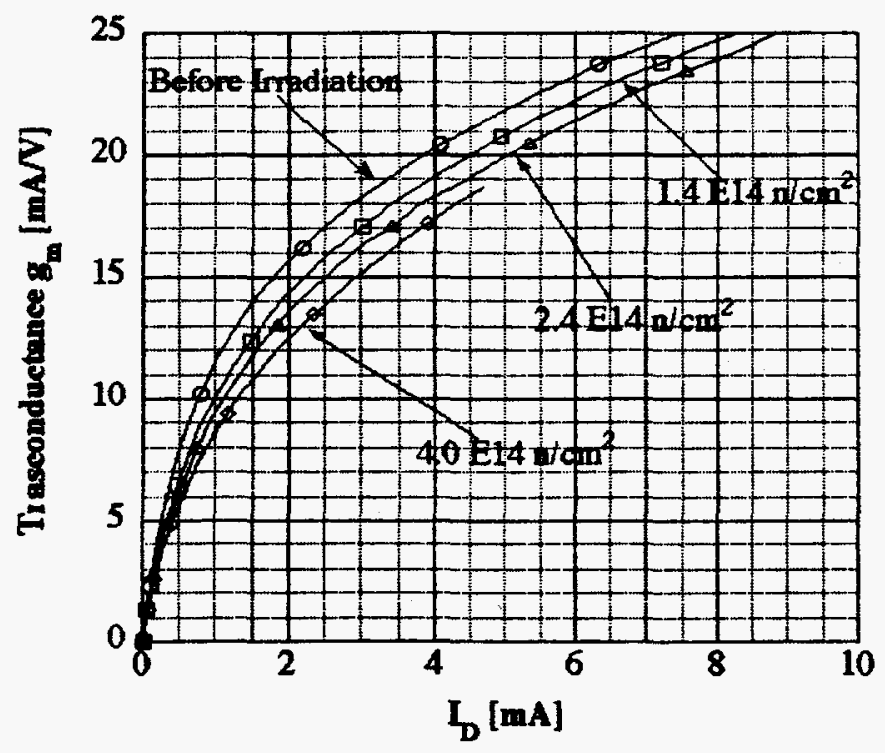

Fig. 5 Trasconductance for a Si-NJFET (Interfet) as a function of neutron fluence. The device parameters are: $W / L=3350 / 3, V_{D S}=2.5 \mathrm{~V}$. 


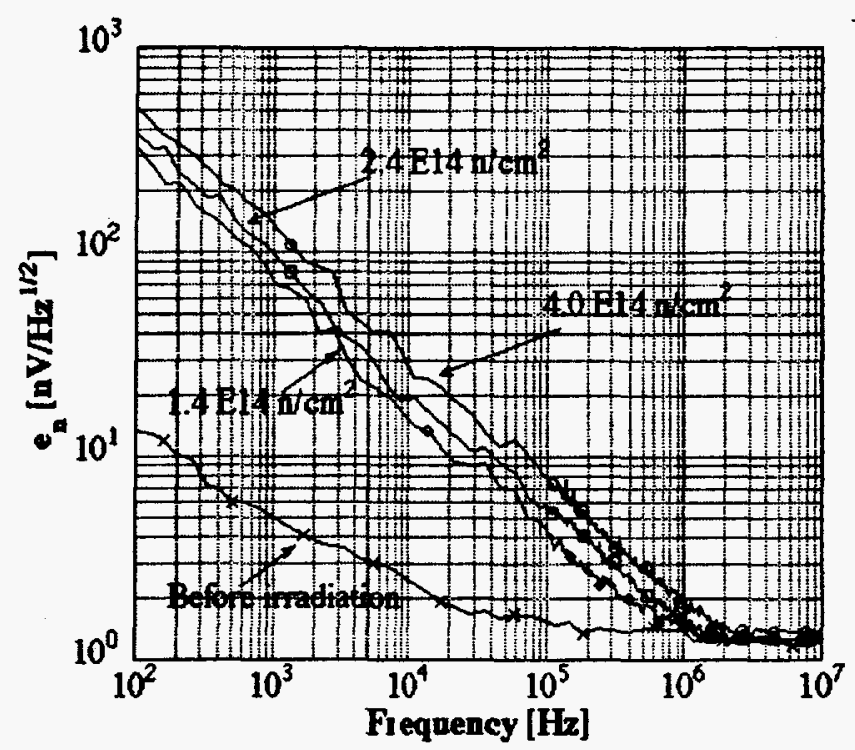

Fig. 6 Series noise spectral density at 98K for a Si-NJFET (Interfet) as a function of neutron fluence. More measurements were performed at high frequency to obtain a better resolution. The device parameters are: $\mathrm{W} / \mathrm{L}=3350 / 3, \mathrm{~V}_{D S}=2.5 \mathrm{~V}, \mathrm{I}_{D}=1 \mathrm{~mA}$.

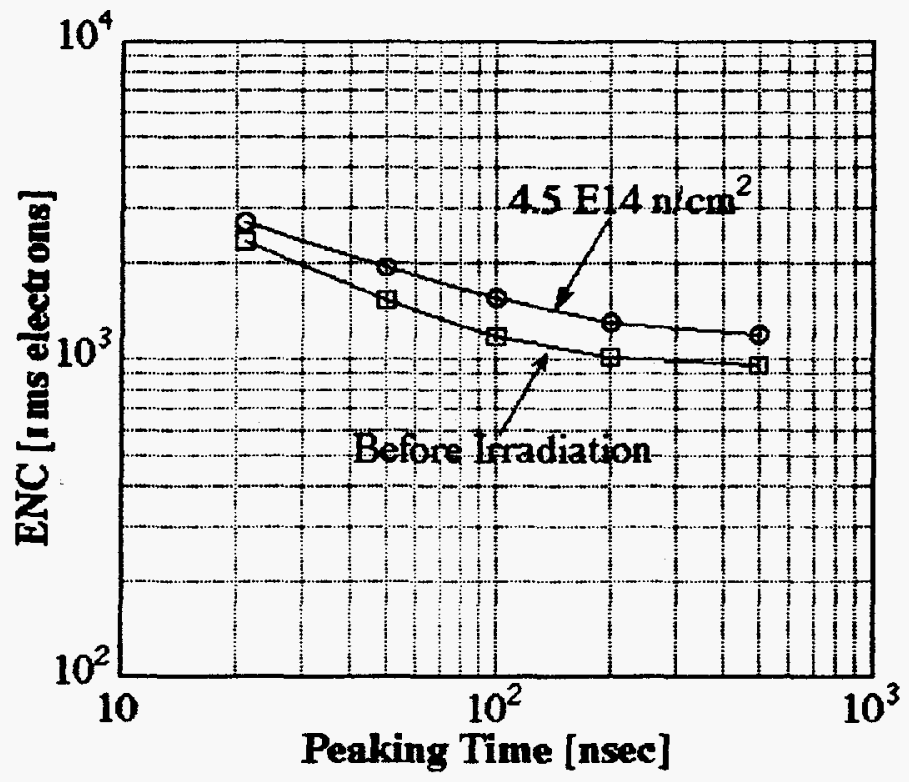

Fig. 7 Equivalent Noise Charge for GaAs preamplifier at $87 \mathrm{~K}$ before and after the exposure to a total fluence of $4.5 \mathrm{E} 10^{14} \mathrm{n} / \mathrm{cm}^{2}$.

\section{B. GaAs MESFETs}

GaAs Mesfets are less affected by neutron irradiation in comparison to Si-JFETs. The geometrical position of our sample with respect to the highly collimated neutron source allowed a total neutron fluence of $1 \times 10^{14} \mathrm{n} / \mathrm{cm}^{2}$. No significant variations have been observed 
up to this level of fluence, both in noise and in DC characteristics.

In other tests performed at SARA, Grenoble [7], GaAs preamplifiers have been irradiated up to neutron fluence of to $4.5 \times 10^{14} \mathrm{n} / \mathrm{cm}^{2}$ at $87 \mathrm{~K}$ [8]. The preamplifiers were able to withstand the test and have shown a noise increase of about $20 \%$ at $90 \mathrm{~K}$ at $40 \mathrm{nsec}$ peaking time as shown in Fig. 7.

\section{Annealing}

No annealing has been observed at cryogenic temperatures over a period of few months from the conclusion of the neutron exposure for the three different technologies.

No thermal cycling has been performed yet.

\section{Conclusions}

The device noise has been identified as the parameter most sensitive to radiation for all the technologies under study.

No variations in the DC properties have been observed for both technologies against ionizing radiation. Si-JFETs devices show a DC characteristic variation at very high neutron fluences.

Both Si-JFETs and GaAs devices have been proved able to operate in the experimental conditions foreseen for fast calorimetry at hadron colliders. Si-JFETs are less sensitive to ionizing radiation than GaAs MESFETs. GaAs Mesfets are less sensitive to neutron irradiation than Si-JFETs.

\section{References}

[1] V. Radeka, S. Rescia, "Speed and Noise Limits in Ionization Chamber Calorimeters", NIM A265 (1988), pp 228-242

[2] ATLAS: Letter of Intent. CERN/LHCC/92-4, LHCC/12

[3] GEM: Technical Design Report, GEM-TN-93-262, SSCL-SR-1219, April 30, 1993

[4] D.E. Groom, Editor, "Radiation Levels in the SSC Interaction Regions", SSC-SR1033

[5] G.H.R. Kegel, "Fast Neutron Generation with a Type CN Van De Graaff Accelerator", NIM B40-41 (1989), pp 1165-1168

[5] K. Kandiah, M.O. Deighton, F.B. Whiting, "A Physical Model for Random Telegraph Signal Currents in Semiconductor Devices", J.Appl.Phys. 66 (2), 15 July 1989

[6] K. Kandiah, "Improvements in Low Frequency Noise of MOSFETs For Front End Amplifiers", NIM A288 (1990), pp 150-156

[7] J. Collot et al., "SARA, a Neutron Irradiation Station Dedicated to Large Hadron Collider Detector Design", Proc. of the IV International Conference on Calorimetry in High Energy Physics, Sept. 19-25, 1993, La Biodola, Isola d'Elba, Italy 
[8] D. V. Camin, "Radiation Resistance of Front-End Electronics" for Liquid Argon Calorimetry at LHC", Proceedings of the IV International Conference on Calorimetry in High Energy Physics, September 19-25, 1993, La Biodola, Isola d'Elba, Italy Calorimetry in High Energy Physics, September 19-25, 1993, La Biodola, Lsola d'Elba, Italy 